\title{
Zwei mit Cyphon kotanus Yoshitomi, 2009 verwandte neue Arten aus Borneo (Coleoptera, Scirtidae) (182. Beitrag zur Kenntnis der Scirtidae)
}

\author{
Mit 12 Figuren
}

BERNHARD KLAUSNITZER ${ }^{1}$

${ }^{1}$ Mitglied des Senckenberg Deutschen Entomologischen Instituts, Lannerstraße 5, 01219 Dresden, Deutschland Published on 2013-12-20

\section{Zusammenfassung}

Aus Borneo werden zwei neue Arten aus der Gattung Cyphon PAYkull, 1799 beschrieben und mit C. kotanus Yosнiтомі, 2009 verglichen. Der Bau des 9. Sternit und des Penis geben Veranlassung, die drei Arten in einer besonderen Gruppe innerhalb der Cyphon variabilis-Gruppe zusammenzufassen (Cyphon kotanus-Untergruppe). Eine Bestimmungstabelle wird gegeben.

\section{Summary}

Two new species of the genus Cyphon PAYkull, 1799 are described from Borneo and are compared with C. kotanus Yosнiтомі, 2009, also occurring on Borneo. Based on the configuration of the 9th sternite and the penis, the erection of a group of its own for the three species appears justified (Cyphon kotanus-Subgroup). An identification key is provided.

\section{Einleitung}

Yosнiтомі (2009) beschrieb Cyphon kotanus aus Malaysia (Borneo, Sabah) und ordnete sie in die Cyphon variabilis-Gruppe ein. Er wies auf Besonderheiten hin, die diese Art innerhalb der Gruppe von anderen Arten abgrenzen und ihr eine isolierte Stellung verleihen (die genannten Merkmale finden sich auch bei den beiden neuen Arten):

- Distalabschnitt des 9. Sternit seitlich verbreitert, mit einem Paar nach hinten gerichteter Fortsätze (Fig. 1, 2, 7, 8).

- Penis mit seitlich am Ende der Pala entspringenden Parameroiden (Fig. 6, 12)
Bei der Untersuchung von Material aus dem Naturhistoriska Riksmuseet, Stockholm wurden zwei neue Arten entdeckt, die die gleichen Fundortetiketten tragen (!). Die identische Etikettierung gibt natürlich zu denken, beide Arten unterscheiden sich aber sehr deutlich. Sie ähneln Cyphon kotanus in wesentlichen Merkmalen.

Für die weitere Bearbeitung der Gattung Cyphon ist es hilfreich, ähnliche Arten zu Gruppen zusammenzufassen, wobei die gemeinsamen Merkmale abgeleiteten Charakter haben sollten. Es wird deshalb eine Cyphon kotanus-Untergruppe auf der Grundlage der genannten Merkmale definiert, die als apomorph angesehen werden können. 


\section{Beschreibungen neuer Arten}

\subsection{Cyphon sinuatosternalis n. sp.}

Holotypus: $o^{\star}$, O. Borneo, Mjöberg / Mt. Tibang, 1,700 m. In coll. Naturhistoriska Riksmuseet Stockholm.

Körperlänge (Länge Pronotum + Elytre) 2,46 mm. Körper oval (Körperlänge/maximale Körperbreite $=1,54$ ).

Kopf gelbbraun, dicht und fein punktiert, hell behaart. Labrum, Mandibeln, Maxillarpalpen und Labialpalpen hellbraun. Abstand zwischen den Innenrändern der Augen 0,48 mm. Labrum dicht behaart, Seiten gebogen. Erstes Antennenglied vorn innen mit einer Kante, braun, übrige Antennenglieder hellbraun, drittes deutlich schmaler als das zweite. Länge 1 . Antennenglied $0,11 \mathrm{~mm} ; 2 \cdot 0,10 \mathrm{~mm} ; 3 \cdot 0,07 \mathrm{~mm} ; 4 \cdot 0,11 \mathrm{~mm}$ (übrige Glieder nicht erhalten).

Pronotum rotbraun, innerhalb der Hinterecken sehr schwach eingeschweift, fein und dicht punktiert, Punktzwischenräume glatt (100fache Vergrößerung), dicht hell behaart. Maximale Breite 0,95 mm; Länge entlang der Mittellinie 0,46 mm. Scutellum gelbbraun, hell behaart.

Elytren einfarbig hellbraun, ohne Rippen, dicht und fein punktiert, Punktzwischenräume glatt (100fache Vergrößerung). Elytren mit heller, nach hinten gerichteter Behaarung. Länge zwischen Schulter und Apex 2,00 mm; Breite einer Elytre in der Mitte 0,80 mm.

Beine hellbraun. 3.-7. Sternit braun, 3. Sternit etwas dunkler, Vorder- und Hinterrand schwarzbraun. Sternite nach hinten gerichtet hell behaart. 7. Sternit hinten gleichmäßig gebogen, maximale Breite $0,84 \mathrm{~mm}$; maximale Länge in der Mitte 0,25 mm.

9. Sternit (Fig. 1) aus einem vorn gerundeten schmalen, parallelseitigen Stab bestehend, der sich hinten zu einer Platte erweitert. Der Stab ist in der Mitte verstärkt. Die Platte läuft hinten in zwei spitz endende, zahnartige Fortsätze aus, die gerade nach hinten gerichtet sind. An der Basis der Zähne befindet sich eine gebogene Querlinie (Fig. 2, Pfeil). Zwischen den Zähnen ist die Platte hinten tief eingebuchtet. Gesamtlänge des 9. Sternit 0,56 mm; Breite der Platte $0,13 \mathrm{~mm}$; Breite der parallelen Basis $0,06 \mathrm{~mm}$.

Das 8. Tergit (Fig. 3) besteht nur aus den Bacilla lateralia, die Platte ist völlig reduziert. Der basale Teil wird vom distalen Teil wie von einer Hülse umgeben, eine Struktur, die in der Cyphon variabilis-Gruppe weit verbreitet ist (NyHolm 1969). Der distale Teil ist mit einer Skulptur aus länglichen Mulden und gebogenen linienartigen Erhebungen bedeckt (Fig. 4). Länge 0,75 mm; Breite $0,02 \mathrm{~mm}$. Das 9 . Tergit besteht aus fast geraden
Bacilla lateralia und einem schwach sklerotisierten Rest der Platte. In der Mitte sind die Bacilla lateralia schwach miteinander verbunden. Länge der Bacilla lateralia $0,62 \mathrm{~mm}$.

Tegmen (Fig. 5) mit einem schmalen lanzettförmigen Kapulus, der seitlich verstärkt ist. Es schließen sich relativ schmale Parameren an (der Distalabschnitt der Parameren ist nur teilweise erhalten, sodass deren Bau nicht im Ganzen beschrieben werden kann). Gesamtlänge des Tegmen 0,28 mm; maximale Breite 0,20 mm.

Penis (Fig. 6) mit einer vorn gebogenen, nach hinten gleichmäßig verbreiterten Pala von annähernd dreieckiger Form, die am Rand verstärkt ist. An den Außenseiten schließen sich lange, zunächst parallel verlaufende, dann nach innen gebogene schmale Parameroide an. Gesamtlänge des Penis $0,52 \mathrm{~mm}$; maximale Breite $0,22 \mathrm{~mm}$; Länge der Pala 0,36 mm; Länge der Parameroide ohne Berücksichtigung der Krümmung 0,17 mm.

Weibchen unbekannt.

Areal: Cyphon sinuatosternalis n. sp. ist bisher nur vom Fundort des Holotypus bekannt. Bei dem Sammler dürfte es sich um ERIC GeORg MjöBerg (geb. 1882) handeln. Er sammelte 1922/1927 auf Borneo.

Derivatio nominis: Der Artname „sinuatosternalis“ setzt sich aus "sinuatus“ (= ausgebuchtet, lat.) und „sternum“ (lat.) zusammen und weist auf die tiefe Einbuchtung des Hinterrandes des 9. Sternit hin.

\subsection{Cyphon labratus n. sp.}

Holotypus: $ð^{\star}$, O. Borneo, Mjöberg / Mt. Tibang, 1,700 m. In coll. Naturhistoriska Riksmuseet Stockholm.

Körperlänge (Länge Pronotum + Elytre) 1,93 mm. Körper länglich oval (Körperlänge/maximale Körperbreite $=1,38)$.

Kopf braun, nach vorn heller werdend, dicht punktiert, hell nach vorn gerichtet behaart. Antennen nicht sichtbar (Exemplar in schlechtem Erhaltungszustand, ein Umpräparieren ist nicht angezeigt).

Pronotum weißbraun, innerhalb der Hinterecken annähernd gerade, fein und dicht punktiert, dicht hell behaart. Maximale Breite 0,75 mm; Länge entlang der Mittellinie $0,33 \mathrm{~mm}$. Scutellum rotbraun.

Elytren einfarbig braun, ohne Rippen, dicht und fein punktiert, mit heller, nach hinten gerichteter Behaarung. Länge zwischen Schulter und Apex 1,60 mm; Breite einer Elytre in der Mitte $0,70 \mathrm{~mm}$. 
Beine hellbraun. 3.-7. Sternit hellbraun. 7. Sternit hinten etwas abgestutzt. Maximale Breite $0,71 \mathrm{~mm}$; maximale Länge in der Mitte 0,25 mm.

9. Sternit (Fig. 7) aus einem schmalen, parallelseitigen Stab bestehend, der sich hinten zu einer breiten Platte erweitert. Der Stab ist in der Mitte verstärkt. Die Platte läuft hinten in zwei spitze zahnartige Fortsätze von $0,06 \mathrm{~mm}$ Länge aus, die etwas nach außen gerichtet sind (Fig. 8). An der Basis der Zähne befindet sich eine gebogene Querlinie. Daneben befindet sich außen jederseits ein kleines Feld von sieben Sinnesporen (Fig. 8, Pfeil) und zwei schmale kurze Borsten. Unterhalb der Zähne liegt ein breiter, lappenartig abgesetzter Teil der Platte, der hinten schwach eingebuchtet ist. Gesamtlänge des 9. Sternit 0,42 mm; Breite der Platte 0,17 mm; Breite der parallelen Basis $0,03 \mathrm{~mm}$.

Das 8. Tergit (Fig. 9) besteht nur aus den isolierten Bacilla lateralia, die Platte ist völlig reduziert. Der basale Teil wird vom distalen Teil wie von einer Hülse umgeben. Der distale Teil ist mit einer Skulptur aus länglichen Mulden und gebogenen, erhabenen Linien bedeckt (Fig. 10). Länge $0,82 \mathrm{~mm}$; Breite $0,025 \mathrm{~mm}$. Das 9 . Tergit besteht aus geraden Bacilla lateralia und einer sehr schwach sklerotisierten Platte. Länge der Bacilla lateralia 0,70 mm.

Tegmen (Fig. 11) mit einem schmalen lanzettförmigen Kapulus und breiten Parameren, die mit zahlreichen dünnen Borsten bedeckt sind. Gesamtlänge des Tegmen $0,26 \mathrm{~mm}$; maximale Breite 0,21 $\mathrm{mm}$.

Penis (Fig. 12) mit einer vorn breit gebogenen, nach hinten erweiterten Pala mit einem geschwungenen Außen- rand. Die Pala ist am Rand verstärkt. An den Außenseiten schließen sich hinten gerade, breite, lappenförmige Parameroide an, die an ihren Innenseiten herausgehobene, faltenartige Sklerotisierungen aufweisen (Fig. 12, Pfeile). Gesamtlänge des Penis $0,34 \mathrm{~mm}$; maximale Breite der Pala 0,18 mm; Länge der Pala 0,23 mm; Länge der Parameroide $0,11 \mathrm{~mm}$.

Weibchen unbekannt.

Areal: Cyphon labratus n. sp. ist bisher nur vom Fundort des Holotypus bekannt.

Derivatio nominis: Der Artname "labratus" ist von „labrum“ (= Lippe, lat.) abgeleitet und bezieht sich auf die Form des Hinterrandes des 9. Sternit.

\section{Differentialdiagnose und Bestimmungs- tabelle für die bisher bekannten Arten der Cyphon kotanus-Untergruppe}

Abgrenzung und Vergleich der beiden neuen Arten untereinander und mit Cyphon kotanus Yosнiтомі, 2009 erfolgen in der Bestimmungstabelle. Die auffallende Skulptur des Distalabschnittes der Hemitergite des 8. Tergit (Fig. 4, 10) wird in der Beschreibung von C. kotanus nicht erwähnt.

1 Parameroide schmal (Fig. 6). Basis des 9. Sternit breiter (0,06 mm), distale Platte weniger abgesetzt (Breite Platte/ Breite basaler $\mathrm{Stab}=2,17)$. Die Zähne sind gerade nach hinten gerichtet. Dazwischen ist der Hinterrand der Platte tief eingebuchtet (Fig. 2). Körperlänge 2,46 mm. Borneo. sinuatosternalis $\mathrm{n} . \mathrm{sp}$

$1^{*}$ Parameroide breit, lappenförmig (Fig. 12). Basis des 9. Sternit schmaler (0,01-0,03 mm), distale Platte stärker abgesetzt (Breite Platte/Breite basaler Stab = 5,67-14,0). Bau der Platte des 9. Sternit anders (Fig. 7, 8).

2 Parameroide gerade, schwach nach außen gerichtet (Fig. 12), nicht winkelförmig. Pala dazwischen nicht vorstehend, nicht deutlich abgesetzt. An den Innenseiten der Parameroide befinden sich herausgehobene faltenartige Sklerotisierungen. Platte des 9. Sternit etwas schmaler (Breite Platte/Gesamtlänge 9. Sternit =0,40). Zähne an der Platte des 9. Sternit ohne kurze Borsten an der Spitze, etwas nach außen gerichtet. Neben der Basis befindet sich außen jederseits ein kleines Feld von sieben Sinnesporen (Fig. 8, Pfeil). Der unterhalb der Zähne gelegene breite, lappenartig abgesetzte Teil der Platte ist hinten schwach eingebuchtet. 9. Tergit mit einer schwach sklerotisierten Platte. Körperlänge 1,93 mm. Borneo. labratus n. sp.

2* Parameroide winkelförmig, proximal nach außen, distal nach innen gerichtet. Pala dazwischen etwas vorstehend, in der Mitte seicht eingebuchtet. Distale Enden der Parameroide ohne herausgehobene, nach innen gerichtete Sklerotisierungen. Platte des 9. Sternit etwas breiter (Breite Platte/Gesamtlänge 9. Sternit =0,51). Zähne an der Platte des 9. Sternit jeweils mit einer kurzen Borste an der Spitze. Sinnesporen sind nicht vorhanden, der Hinterrand ist nicht abgesetzt. 9. Tergit völlig ohne Platte, es sind nur die Bacilla lateralia vorhanden. Körperlänge 2,43 mm. Borneo. kotanus Yoshiтomi, 2009 


\section{Dank}

Herrn Dr. Bert VikLund, Naturhistoriska Riksmuseet Stockholm, danke ich sehr herzlich für die großzügigen Möglichkeiten zur Bearbeitung der Scirtidae aus den Sammlungen des Museums.

\section{Literatur}

Nyholm, T. 1969: Über Bau und Funktion der Kopulationsorgane bei den Cyphones (Coleoptera, Helodidae). Studien über die Familie Helodidae. X. - Entomologisk Tidskrift 90: 233-271.

Yoshiтомi, H. 2009: Scirtid Beetles (Coleoptera, Scirtidae) of the Oriental Region, Part 10. New Species and New Records of Cyphon variabilis Species-Group. Elytra, Tokyo 37 (1): 87-97.
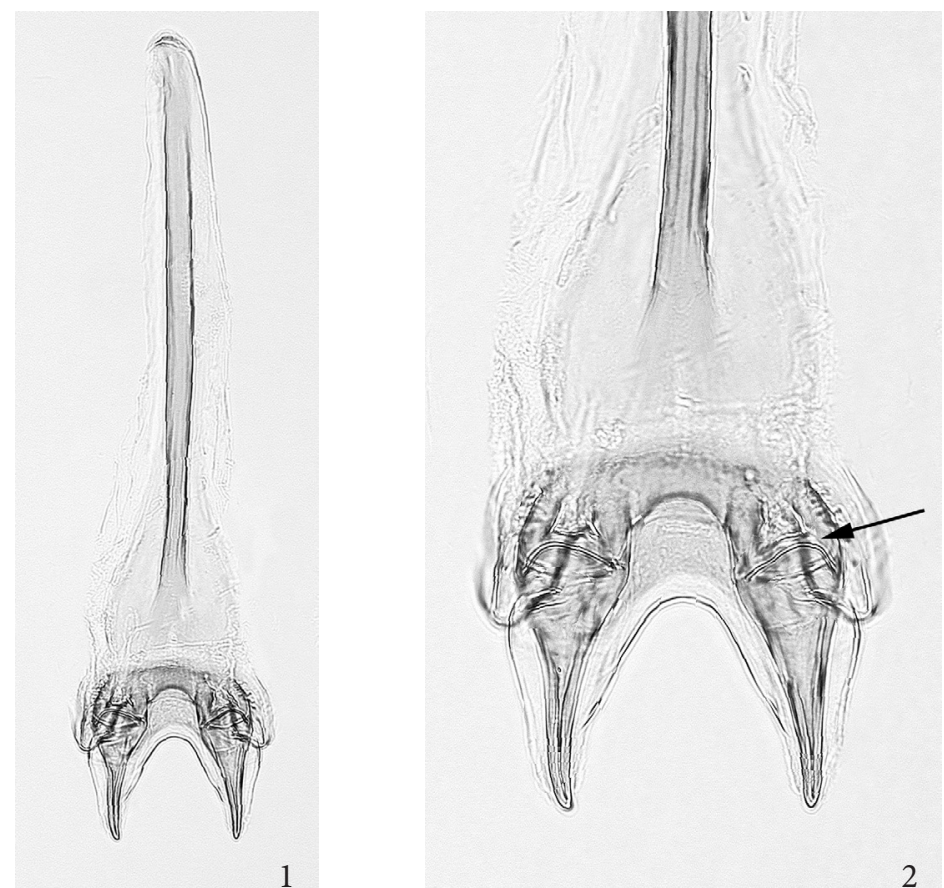

2
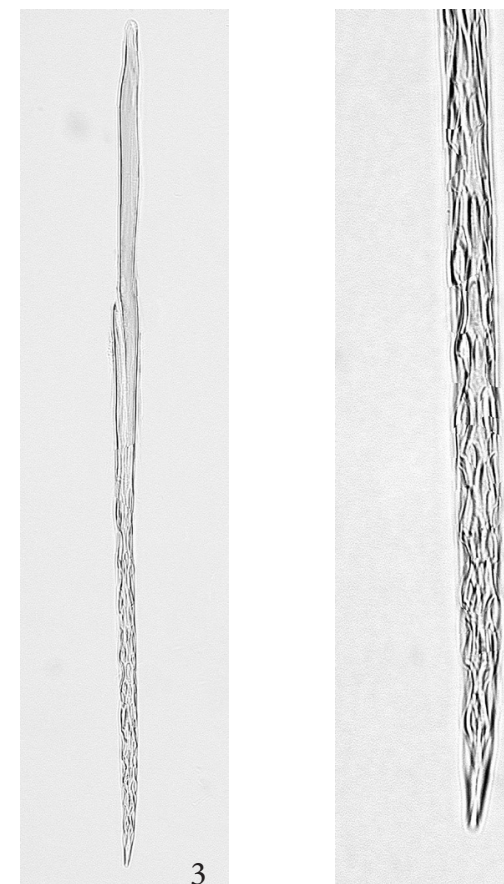

Fig. 1-4: Cyphon sinuatosternalis n. sp. 1: 9. Sternit; 2: 9. Sternit, Distalabschnitt; 3: 8. Tergit; 4: 8. Tergit, Distalabschnitt.
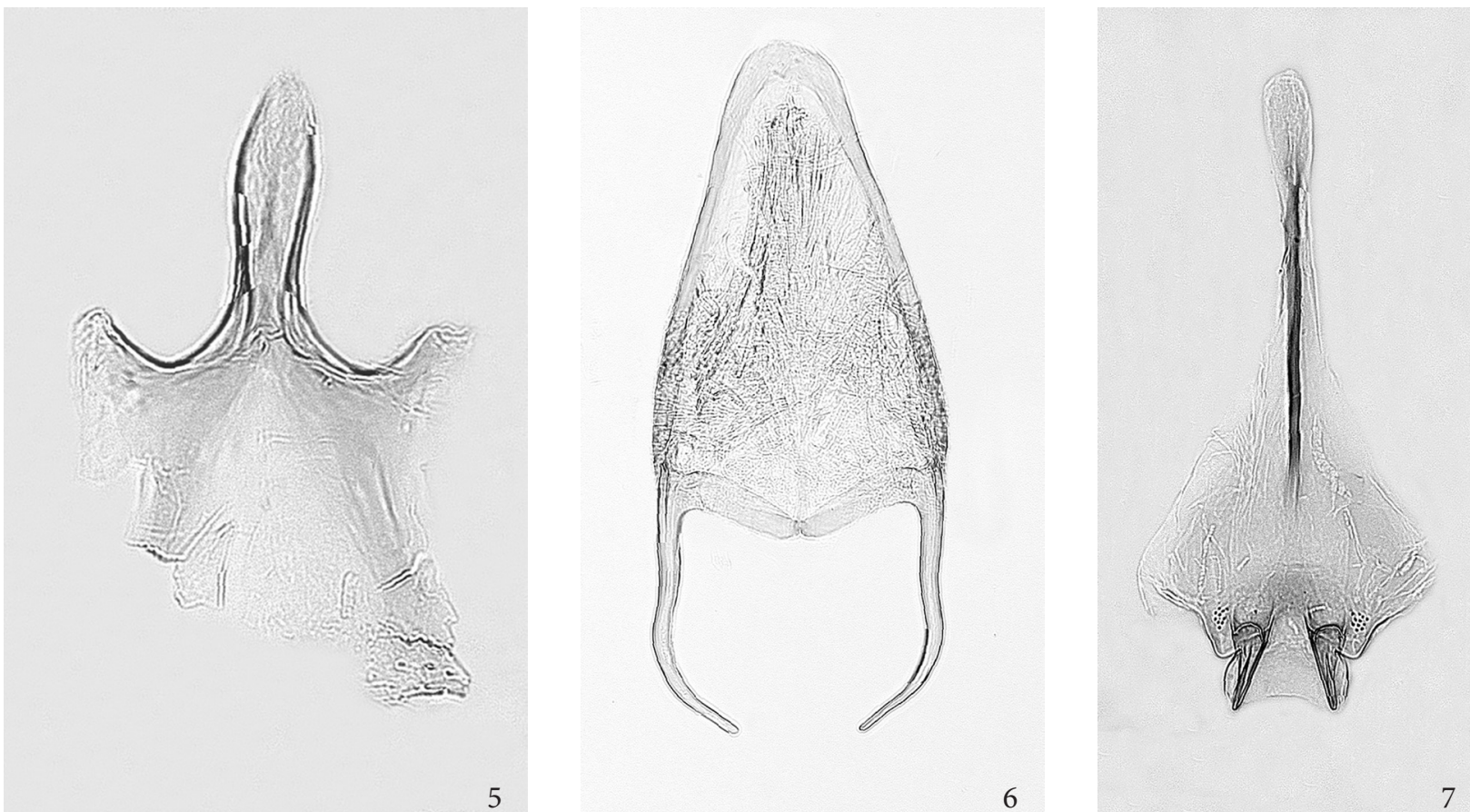

Fig. 5-7: Cyphon sinuatosternalis n. sp. 5: Tegmen, vorderer Abschnitt; 6: Penis; 7: Cyphon labratus n. sp., 9. Sternit. 

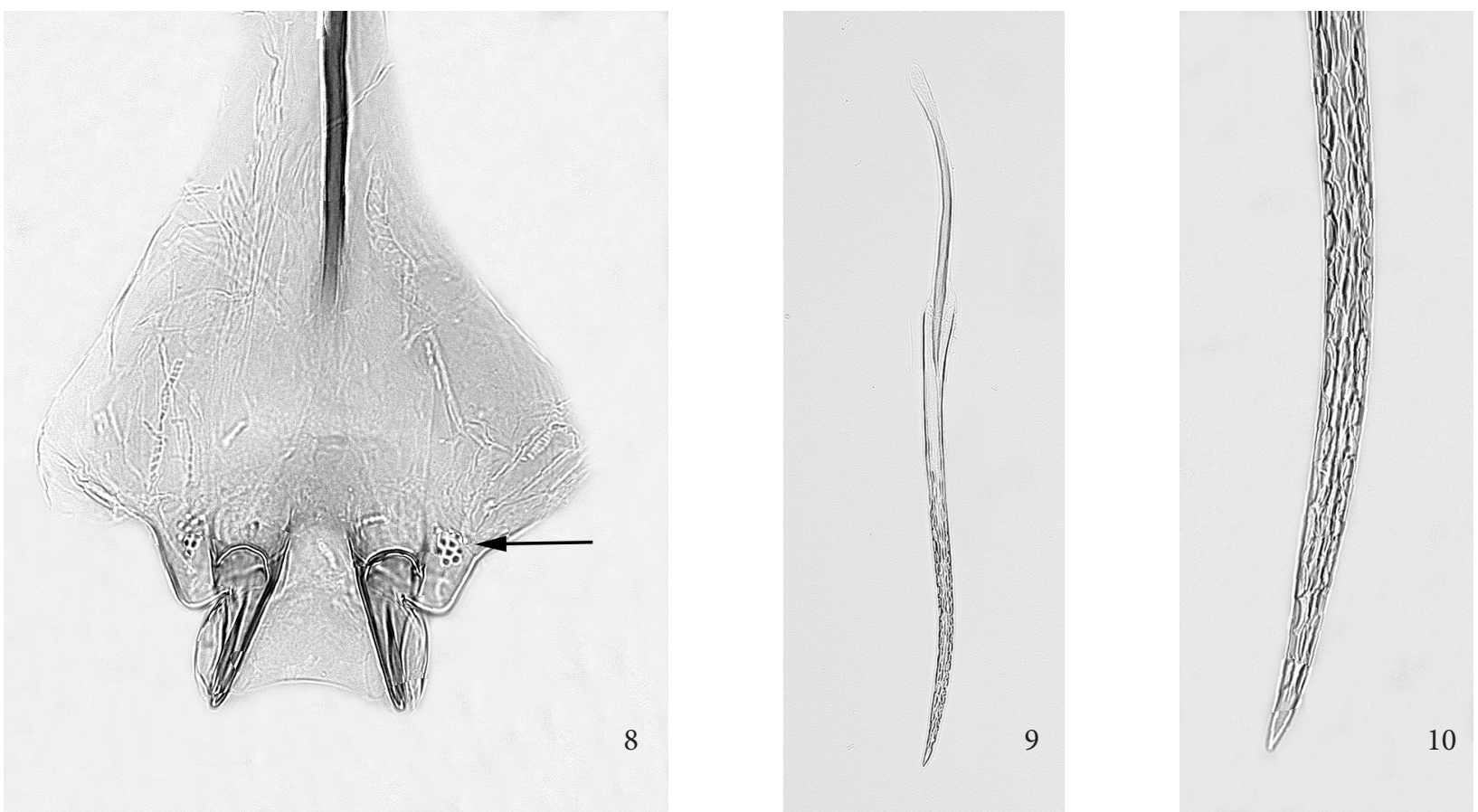

Fig. 8-10: Cyphon labratus n. sp. 8: 9. Sternit, Distalabschnitt; 9: 8. Tergit; 10: 8. Tergit, Distalabschnitt.
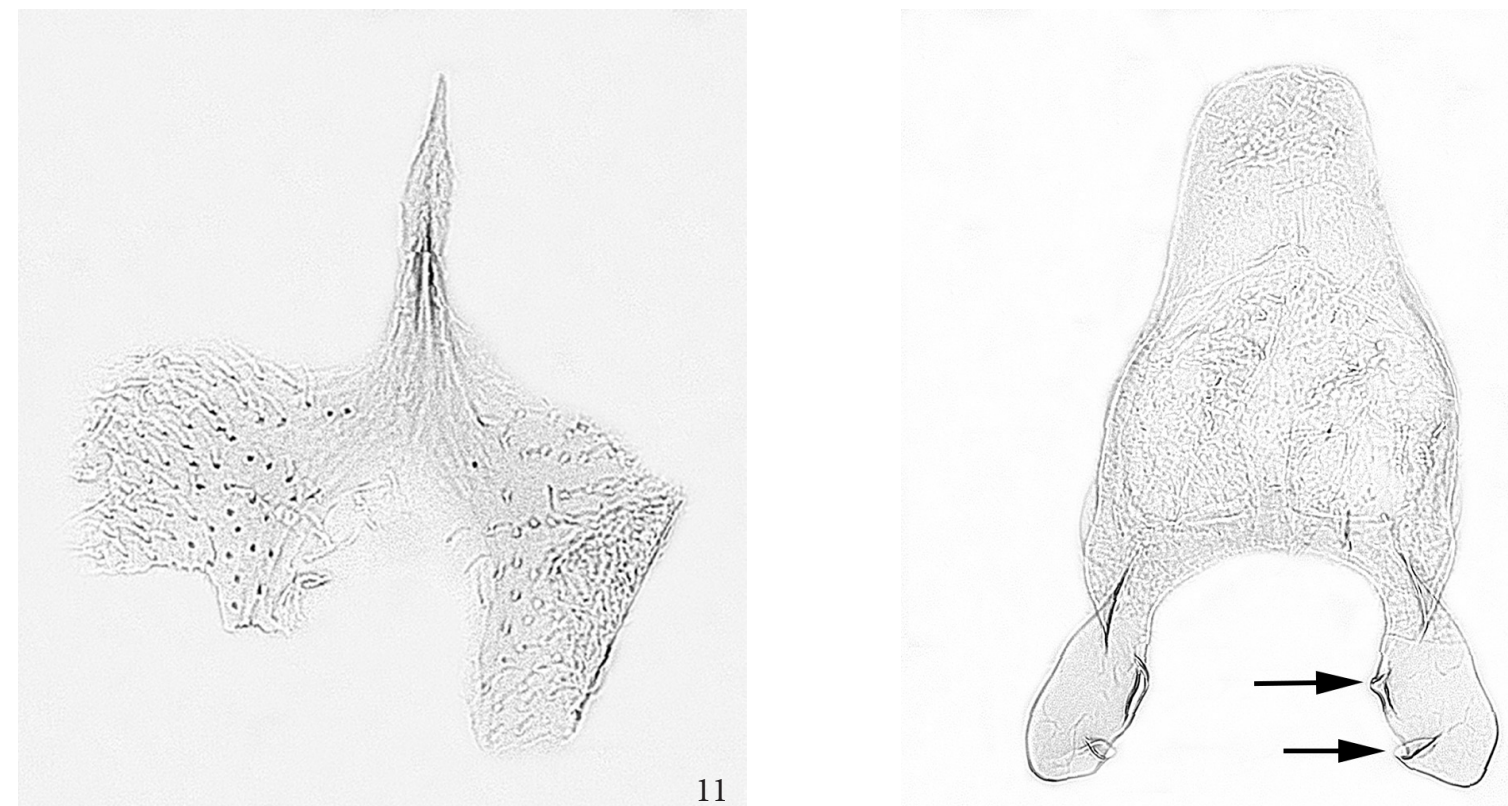

Fig. 11-12: Cyphon labratus n. sp. 11: Tegmen; 12: Penis. 